\title{
Menopause
}

\section{Ekanayake $C^{1}$}

${ }^{1}$ Consultant Obstetrician and Gynaecologist and Senior Lecturer, Department of Clinical Sciences, Faculty of Medicine, General Sir John Kotelawala Defense University.

Email-cdekanayake2000@yahoo.com

1. Regarding menopause

a. Vasomotor symptoms are present in $75 \%$ of women going through menopause.

b. Estrogen alone preparations have a beneficial effect on high-density lipoproteins (HDL) and low-density lipoprotein (LDL).

c. A significant amount of women with fracture neck of femur die within the first year.

d. If the duration of treatment of Estrogen exceeds 1 year, it has to be under specific individualized indications with intense follow-up and monitoring.

e. All women should have screening for BRCA gene mutation even if there is a negative history for breast and ovarian cancer.

2. The following are contraindications for hormone replacement treatment (HRT)
a. Diabetes mellitus
b. Past history of breast cancer
c. Migraine
d. Past history of deep vein thrombosis
e. Essential hypertension

3. A 55-year old woman with hot flushes and night sweats presents to the gynaecology clinic. She underwent a mastectomy and radiotherapy for breast cancer 3-years back. She has a strong family history of osteoporosis. What is the best option to manage her symptoms?
a. Hormone replacement treatment (HRT)
b. Raloxifene
c. Tamoxifen
d. Norethisterone
e. Transdermal HRT

4. A 60-year old woman with a documented history of Transient Ishaemic Attacks (TIA) complaints of vasomotor symptoms. What is the best treatment option,
a. Hormone replacement treatment (HRT)
b. Transdermal HRT
c. Vaginal Estrogen
d. Tamoxifen
e. Phyto-Estrogen

5. A45-year old woman with two children complains of headache, sweating and hot flushes. She had a hysterectomy five years back for menorrhagia. The general and gynaecological examination findings were normal. What is the most appropriate treatment regimen?
a. Vitamin E
b. Norethisterone
c. Estrogen and progestogen HRT
d. Combined oral contraceptive pill
e. Estrogen only HRT

\section{$\underline{\text { References }}$}

1. Patterson M. Menopause and hormone replacement therapy. In: Luesley DM, Baker PN, editors. Obstetrics and Gynaecology: An evidence-based text for MRCOG. 2nd ed. London: Hodder Arnold; 2010. 
2. Luesley DM, Baker PN, editors. MCQs and Short Answer Questions for MRCOG. An aid to revision and self-assessment. 1st ed. London: Hodder Arnold; 2004.

3. Perera H. Ekanayake C. Menopausal hormone therapy with Estrogen and progestogen. Sri Lanka Journal of Menopause. 2019; $1(1): 5-12$.

\section{$\underline{\text { Answers }}$}

1. All true

2. FTFTT

3. D

4. B

5. E

* Table 01 shows a summary of menopausal treatment options 


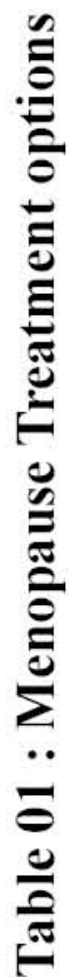

\begin{tabular}{|c|c|c|c|c|c|c|}
\hline 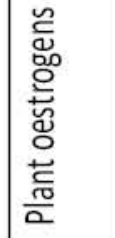 & 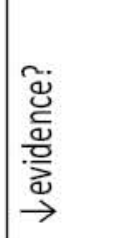 & $\leftarrow$ & $\rightarrow$ & & & \\
\hline 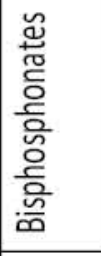 & & & $\rightarrow$ & & & \\
\hline 蕊 & $\rightarrow$ & & & & & \\
\hline 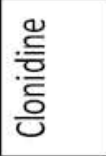 & 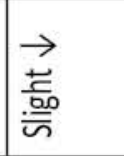 & & & & & \\
\hline 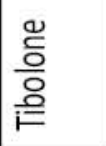 & $\rightarrow$ & & & & & \\
\hline 总 & $\leftarrow$ & $\rightarrow$ & $\rightarrow$ & & & \\
\hline 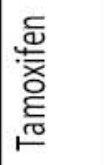 & $\leftarrow$ & $\rightarrow$ & $\rightarrow$ & & $\leftarrow$ & $\leftarrow \leftarrow$ \\
\hline 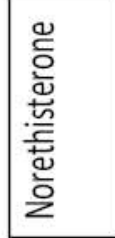 & $\rightarrow$ & $\leftarrow$ & . & . & $\rightarrow$ & \\
\hline 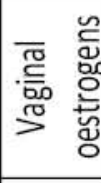 & & . & . & & & \\
\hline 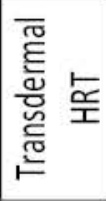 & $\rightarrow$ & $\leftarrow$ & . & 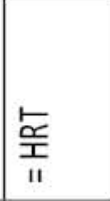 & & . \\
\hline 点 & $\rightarrow$ & $\leftarrow$ & $\rightarrow$ & 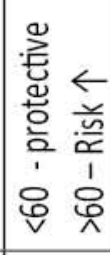 & $\begin{array}{cc} & \\
\overleftarrow{1} & \vdots \\
0 & \vdots \\
0 & 0\end{array}$ & $\leftarrow$ \\
\hline & 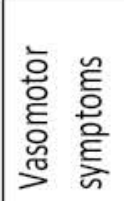 & 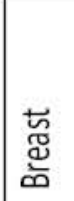 & 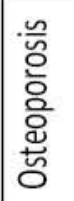 & 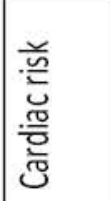 & 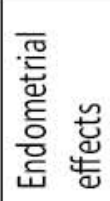 & 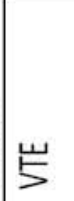 \\
\hline
\end{tabular}


